\title{
A Study of Six Degrees of Freedom Welding Robot Path Planning Based on Genetic Algorithms
}

\author{
Haiming Shen ${ }^{1, a^{*}}$, Qian Kang ${ }^{2, b}$ and Xiaofeng Yuan ${ }^{3, c}$ \\ ${ }^{1}$ ChongQing University of Arts and Sciences, China \\ ${ }^{2}$ Chongqing Water Resources And Electric Engineering College, China \\ ${ }^{3}$ Yongchuan Middle School, China \\ AShenhaiming13@163.com, b43721998@qq.com, c283225283@qq.com
}

Keywords: Genetic algorithms; six degrees of freedom; path planning; multi-objective optimization.

\begin{abstract}
In this paper, modeling is planned on the basis of characteristics of six degrees of freedom welding robot. According to the characteristics of the genetic algorithm, the modeling take the advantage of the most representative weighting method of multi-objective optimization design method to weigh all the performance requirements and determine the fitness function, and to plan an optimal trajectory capable of keeping the arm of welding robot from multiple obstacles and meeting the requirements of exercise time, track length, kinematics and dynamics at the same time.
\end{abstract}

\section{Introduction}

Six degrees of freedom welding robot path planning method consist of local path planning like artificial potential field method and fuzzy logic method, etc. based on sensor and global path planning like visibility graphs, free space method, optimal control method, topological method[1], etc. based on environmental information. As it is impossible for local path planning to realize overall obstacle avoidance, while traditional overall obstacle avoidance method is of low computational efficiency and not suitable for high-dimensional optimization, genetic algorithm is comparatively optimization one based on intelligent bionic algorithm and able to solve the problems existing in the traditional global avoidance method better.

\section{Overview of Genetic Algorithms}

On the basis of Darwin's theory of evolution, genetic algorithm simulates the evolution of the biosphere nature in computer system which follows survival of the fittest screening mechanism. As a random search method, its main function is to obtain external environment information automatically by the early generation group and gradually accumulate to achieve a global search for the optimal progeny group. It has features of global optimization and parallelism. Due to all these nature of genetic algorithm, it is widely used in calculation of the life sciences, machine signal processing, path planning and other intelligent modern technology calculating.

\section{The modeling of six DOF welding robot}

The structural parameters of six DOF welding robot studied in this paper is shown in Table 1 . 
TABLE I. Joint parameters of six DOF welding robot

\begin{tabular}{|l|l|l|l|l|l|}
\hline $\begin{array}{l}\text { Connec } \\
\text { ting rod } \\
\mathrm{i}\end{array}$ & $\begin{array}{c}\text { variabl } \\
\theta_{i}\end{array}$ & $\alpha_{l-1}$ & $a_{l-1}$ & $d_{i}$ & Variable range $\theta_{i}$ \\
\hline 1 & $\theta_{1}$ & $0^{\circ}$ & 0 & 0 & $-160^{\circ} \sim 160^{\circ}$ \\
\hline 2 & $\theta_{2}$ & $90^{\circ}$ & 0 & $d_{2}$ & $-225^{\circ} \sim 45^{\circ}$ \\
\hline 3 & $\theta_{3}$ & $90^{\circ}$ & $a_{2}$ & 0 & $-45^{\circ} \sim 225^{\circ}$ \\
\hline 4 & $\theta_{4}$ & $90^{\circ}$ & $a_{3}$ & $d_{4}$ & $-110^{\circ} \sim 170^{\circ}$ \\
\hline 5 & $\theta_{5}$ & $90^{\circ}$ & 0 & 0 & $-100^{\circ} \sim 100^{\circ}$ \\
\hline 6 & $\theta_{6}$ & $90^{\circ}$ & 0 & 0 & $-268 \sim 268$ \\
\hline
\end{tabular}

According to the parameters, every connecting rod transformation matrix is available:

$$
\begin{aligned}
& { }^{0} T_{1}=\left[\begin{array}{cccc}
C_{\theta_{1}} & -S_{\theta_{1}} & 0 & 0 \\
S_{\theta_{1}} & C_{\theta_{1}} & 0 & 0 \\
0 & 0 & 1 & 0 \\
0 & 0 & 0 & 1
\end{array}\right]{ }^{1} T_{2}=\left[\begin{array}{cccc}
C_{\theta_{2}} & -S_{\theta_{2}} & 0 & 0 \\
0 & 0 & 1 & d_{2} \\
-S_{\theta_{2}} & -C_{\theta_{2}} & 0 & 0 \\
0 & 0 & 0 & 1
\end{array}\right]{ }^{2} T_{3}=\left[\begin{array}{cccc}
C_{\theta_{3}} & -S_{\theta_{3}} & 0 & a_{2} \\
S_{\theta_{3}} & C_{\theta_{3}} & 0 & 0 \\
0 & 0 & 1 & 0 \\
0 & 0 & 0 & 1
\end{array}\right] \\
& { }^{3} T_{4}=\left[\begin{array}{cccc}
C_{\theta_{4}} & -S_{\theta_{4}} & 0 & a_{3} \\
0 & 0 & 1 & d_{4} \\
-S_{\theta_{4}} & -C_{\theta_{4}} & 0 & 0 \\
0 & 0 & 0 & 1
\end{array}\right]{ }^{4} T_{5}=\left[\begin{array}{cccc}
C_{\theta_{5}} & -S_{\theta_{5}} & 0 & 0 \\
0 & 0 & -1 & 0 \\
S_{\theta_{5}} & C_{\theta_{5}} & 0 & 0 \\
0 & 0 & 0 & 1
\end{array}\right]{ }^{5} T_{6}=\left[\begin{array}{cccc}
C_{\theta_{6}} & -S_{\theta_{6}} & 0 & 0 \\
0 & 0 & -1 & 0 \\
S_{\theta_{6}} & C_{\theta_{6}} & 0 & 0 \\
0 & 0 & 0 & 1
\end{array}\right]
\end{aligned}
$$

Figure 1 is a mechanism motion diagram of six DOF welding robot. The connecting rod structure is like a man's arm, joint movement between connecting rods is equivalent of a man's wrist, elbow and shoulder joints, six rotary joint realizes the six degrees of freedom. The first three joints determine the position of wrist reference point, while the rest three determine the orientation of wrist [2], it has the advantage of flexibility and wide working space.

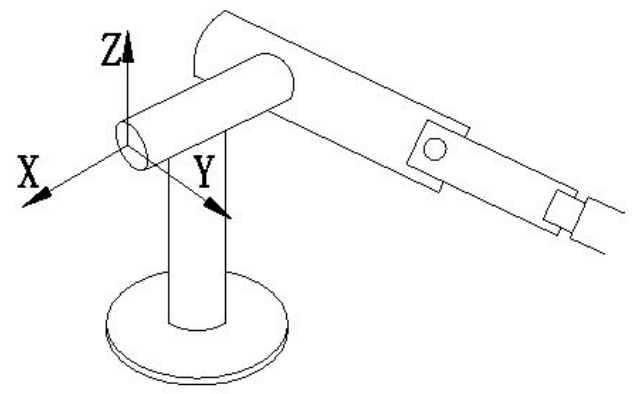

Figure 1. Mechanism diagram of a six DOF welding robot

The equations are an exception to the prescribed specifications of this template. You will need to determine whether or not your equation should be typed using either the Times New Roman or the Symbol font (please no other font). To create multileveled equations, it may be necessary to treat the equation as a graphic and insert it into the text after your paper is styled.

\section{Multi-objective optimization of genetic algorithm}

General path planning deals with single objective optimization problems which mainly involves optimization of the shortest movement time and path of a mechanical arm. However, in actual production process, besides the shortest time and path, other practical problems should be taken into account. In the actual welding process, for example, in order to improve the production efficiency and simply shortening the time of welding process, speed and acceleration of mechanical arm have to be enhanced undoubtedly, which is likely to cause increase of the robot torque of each joint. if such 
increase is beyond the maximum torque, the service life of the mechanical arm shall be affect. The over increment of robot joint angle in collision free trajectory of mechanical arm shall lead to overtime of the end executor and increase of the welding time. These questions contain each other. after integrating the above problems, in order to reflect the reality, the multi-objective optimization realized by genetic algorithm shall help to find the optimal trajectory to balance these problems.

$$
\begin{gathered}
\max \\
\text { S.t }
\end{gathered}
$$

In this function, $\eta$ is weighting factor, weighting factor is set to evaluate the importance of index, it determines the degree of importance. it is a kind of quantitative value indicating judgment orientation of index. For instance, if $f_{1}(x)$ stands for the total time of trajectory, $f_{2}(x)$ the sum of torsion of a mechanical arm during movement, the set value of $\eta_{1}$ and $\eta_{2}$ will affect the balance between the two. If $\eta_{1}$ is bigger than $\eta_{2}$, it indicates that the importance is partial to the total movement time. Importance is influenced by subjective factors, there is no absolute standard of right or wrong, but it is also the reason why the weight can be altered combined with the actual production according to the requirement of valuers.

\section{Steps of the genetic algorithm in path planning}

A. To change the default, adjust the template as follows.

First, $P_{N}$ set of genes is to be set, a set of genes is a trajectory, values of each set of genes is random, namely all parameters of a trajectory are determined, including the Angle, angular velocityand sports time of each joint. In This paper, $P_{N}$ is set as 800 . The more $P_{N}$ value is, the greater the chance to approach the ideal trajectory, meanwhile, the amount of calculation will also increase.

Six degrees of freedom welding robot path planning method consist of local path planning like artificial potential field method and fuzzy logic method, etc. based on sensor and global path planning like visibility graphs, free space method, optimal control method, topological method[3], etc. based on environmental information. As it is impossible for local path planning to realize overall obstacle avoidance, while traditional overall obstacle avoidance method is of low computational efficiency and not suitable for high-dimensional optimization, genetic algorithm is comparatively optimization one based on intelligent bionic algorithm and able to solve the problems existing in the traditional global avoidance method better.

\section{B. To determine the fitness function}

To judge whether the found genome is optimal is to judge whether the gene is the most close to the fitness function. if the error between the two is very small, this set of genes is the optimal solution and namely the end of the program. If the difference is very big, the set of genome has to be deleted and another set of genome should be replenished randomly through the program. $P_{N}$ values are not changed to ensure that early generation groups remain the same. [4]

According to the operating characteristic of mechanical arm, fitness index is defined as follows

$$
f_{G}=f_{b} /\left(\eta_{1} f_{z}+\eta_{2} f_{q}+\eta_{3} f_{1}+\eta_{4} f_{t}\right)
$$

The weighted factor here means demand for the corresponding parameters. Take $\eta_{1}>\eta_{3}$ for example, each joint torque should be ensured less than the maximum one in movement process, and the length of the terminal trajectory is not the shortest. So setting the priority of parameters should ensure that all are the same, or the small one shall lose efficacy. The bigger the result of the fitness function is, the more likely to get the optimal solution.

(1)For interference value, 0 stands for encountering obstacles; 1 avoiding obstacles.

(2) Torque ${ }^{J_{t o}}$ calculation: 


$$
\begin{aligned}
& \tau=M(\theta) \theta^{\prime \prime}+B(\theta)\left[\theta^{\prime} \theta^{\prime}\right]+C(\theta)\left|\theta^{\prime 2}\right|+G(\theta) \\
& f_{t}=\sum_{i=0}^{t_{1} / T+t_{2} / T+t_{3} / T} \sum_{j=1}^{6}\left(\left|\tau_{i, j}-\widehat{\tau}_{j}\right|\right), i f, \tau_{i . j}>\hat{\tau}_{j}
\end{aligned}
$$

In the function:

$M(\theta)$ : Inertia matrix

$B(\theta):$ Coriolis force coefficient matrix

$C(\theta)$ : Centrifugal force coefficient matrix

$G(\theta)$ : Gravity vector set according to the microgravity conditions

$\theta^{\prime}$ is for the former calculation of section function $v_{\theta_{1}}(t), v_{\theta_{2}}(t), v_{\theta_{3}}(t), \theta^{\prime}$ is for $a_{\theta_{1}}(t), a_{\theta_{2}}(t)$, $a_{\theta_{3}}(t)$. joint torque is calculated in each control cycle, therefore, $i$ stands for the $i$ cycle, $j$ the $j$ joint, $\tau$ torque, $\hat{\tau}$ torque limit.

(3) Joint Angle increment and $f_{q}$ calculation:

$$
f_{q}=\sum_{i=0}^{t_{1} / T+t_{2} / T+t_{3} / T} \sum_{j=1}^{6}\left(\left|q_{i, j}-q_{i-1, j}\right|\right)
$$

Among them, $q$ is for the joint Angle value, $i$ the $i$ cycle, $j$ the $j$ joint.

(4)Terminal trajectory length $f_{l}$ calculation:

$$
f_{l}=\sum_{i=0}^{t_{1} / T+t_{2} / T+t_{3} / T}\left[\left(p_{i, x}-p_{i-1, x}\right)^{2}+\left(p_{i, y}-p_{i-1, y}\right)^{2}+\left(p_{i, z}-p_{i-1, z}\right)^{2}\right]^{1 / 2}
$$

Here, $\left(p_{i, x}, p_{i, y}, p_{i, z}\right)$ is the robot end coordinates positioned in the space in the $i$ cycle [4].

\section{The summary}

In line with the requirement of obstacle avoidance for the welding robot in the known environment information space, the multi-objective optimization path planning method based on genetic algorithmin the three-dimensional space is proposed in the study. Meanwhile, the study theoretically optimizes kinematics performance of sis DOF welding robot, and achieves a continuous motion trajectory meeting the requirement of velocity and acceleration by using polynomial function featuring the time of the second order differentiablility[5]. In the study, weighting method is used to weigh the sum of robot joint movement torque, and Angle increment and the importance of terminal trajectory length, and finally get the optimal trajectory making welding robot avoid the space obstacles.

\section{Acknowledgment}

This work was supported financially by the Local Fund of ChongQing Shi Jiaoyu Weiyuanhui (KJ1401107).

\section{References}

[1] Daokui qu, Zhenjun Du, Dianguo Xu, Fang Xue. Mobile robot path planning method research [J]. Journal of robot. 2008, 30 (2) 
[2] Bin $\mathrm{Hu}$. Pouring robot development and kinematics simulation servo system [D]. Nanjing university of science and technology, 2000

[3] Zhixing Chen. Multi-objective genetic algorithm for robot path planning more research [J]. Journal of central south university. 2009

[4] RuoLong Qi, Wei-jia zhou, Tiejun wang. A genetic algorithm based on the space manipulator obstacle avoidance path planning method [J]. Robot, 2014.5 (36) : 263-270

[5] Biyun Xie, Jing Zhao, etc. Based on the rapid expansion of random tree point $7 \mathrm{r}$ manipulator obstacle avoidance for motion planning [J]. Journal of mechanical engineering, 2012, 13 (3). 